\title{
Ultrafast Electric-Field-Driven Control of the Structure of WTe 2 via Terahertz Frequency Light Pulses
}

\author{
Edbert $\mathrm{Sie}^{1}$, Clara Nyby ${ }^{1}$ and Aaron Lindenberg ${ }^{1}$ \\ 1. Stanford University / SLAC National Accelerator Laboratory, Stanford, USA.
}

Tungsten ditelluride is a layered transition-metal dichalcogenide that crystalizes in a distorted hexagonal net with an orthorhombic unit cell. The lack of inversion symmetry in this phase leads to a predicted new topological semimetal with unique optoelectronic properties. Here, we use intense single cycle $\mathrm{THz}$ pulses to trigger a structural deformation in $\mathrm{WTe}_{2}$ and probe its dynamics using femtosecond resolution electron diffraction techniques. We observe large amplitude interlayer shear oscillations at $0.25 \mathrm{THz}$ that occur along the in-plane transition state separating the orthorhombic and monoclinic phases of the material, identified by measuring structure factor modulations of many Bragg reflections. The response scales linearly with the applied $\mathrm{THz}$ field but the initial atomic displacements occur always in the direction towards that of the monoclinic phase, independent of whether the $\mathrm{THz}$ field is applied parallel or antiparallel to this crystallographic direction. We also report on recent results using mid-infrared excitation which show similar structural deformations but of even larger amplitude. This work opens up new possibilities for ultrafast $\mathrm{THz}$ field control over the topological properties of solids.

Measurements were carried out at the SLAC Ultrafast Electron Diffraction (UED) facility [1,2] using relativistic $3 \mathrm{MeV}$ electron bunches, probing single $\sim 10 \mu \mathrm{m}$ x $10 \mu \mathrm{m}$ x $30 \mathrm{~nm}$ flakes of $\mathrm{WTe}_{2}$ exfoliated from a bulk crystal and stamped onto a SiN membrane. The electron beam was incident at normal incidence in a transmission electron diffraction geometry. Measurements were taken first with $800 \mathrm{~nm}$ femtosecond pulses and then subsequently with quasi-single-cycle $\mathrm{THz}$ pulses using both standard $\mathrm{LiNbO}_{3}$-based tilted pulse front and organic crystal optical rectification approaches, with peak applied electric fields $\sim 600 \mathrm{kV} / \mathrm{cm}$. Measurements were also carried out using multicycle mid-infrared photoexcitation with wavelength $\sim 13 \mu \mathrm{m}$.

Fig. 1 shows the time-dependence of several measured Bragg reflections, showing large amplitude oscillatory motion for certain reflections at a frequency of $\sim 0.25 \mathrm{THz}$, matching a known Raman active and IR active mode in $\mathrm{WTe}_{2}$. As shown, whereas reflections of the form (h00) show negligible changes (except for a time-zero streaking effect induced by the interaction of the THz field with the e-beam), reflections with miller index k non-zero exhibit a complex response with certain reflections increasing initially and others decreasing. By measurement of many such reflections we can unambiguously identify the crystallographic axis and initial direction of this shear mode since the starting phase lacks inversion symmetry. This corresponds to motion along the b-axis ([ $\left.\left[\begin{array}{lll}0 & -1 & 0\end{array}\right]\right)$ with displacements corresponding to motion towards that of the centrosymmetric 1T' phase, not a stable phase of the material under equilibrium conditions. This same initial response is induced independent of whether the applied $\mathrm{THz}$ field is applied parallel or antiparallel to the $\left[\begin{array}{lll}0 & -1 & 0\end{array}\right]$ crystallographic axis, with an overall isotropic polarization response. However, the magnitude of the shear amplitude increases linearly with the applied THz field, as also shown in Fig. 1. This is indicative of an effect in which transient in-plane field-driven currents/doping within the polar metallic phase destabilize the orthorhombic $\left(\mathrm{T}_{\mathrm{d}}\right)$ phase of $\mathrm{WTe}_{2}$ and stabilize that of the monoclinic 1T' phase [3]. Similar effects are observed under few cycle mid-infrared photoexcitation with a similar linear-in-field response despite the multicycle excitation in this limit. Because the topological properties of this material are intimately 
connected with its structural properties, this result therefore opens up new possibilities for manipulating the topological properties of Weyl semimetal materials.

References:

[1] S.P. Weathersby et al, Review of Scientific Instruments 86 (2015), p. 073702.

[2] E.M. Mannebach et al, Nano Lett. 15 (2015), p. 6889.

[3] H.-J. Kim et al, Phys Rev B 95 (2017), p. 180101.
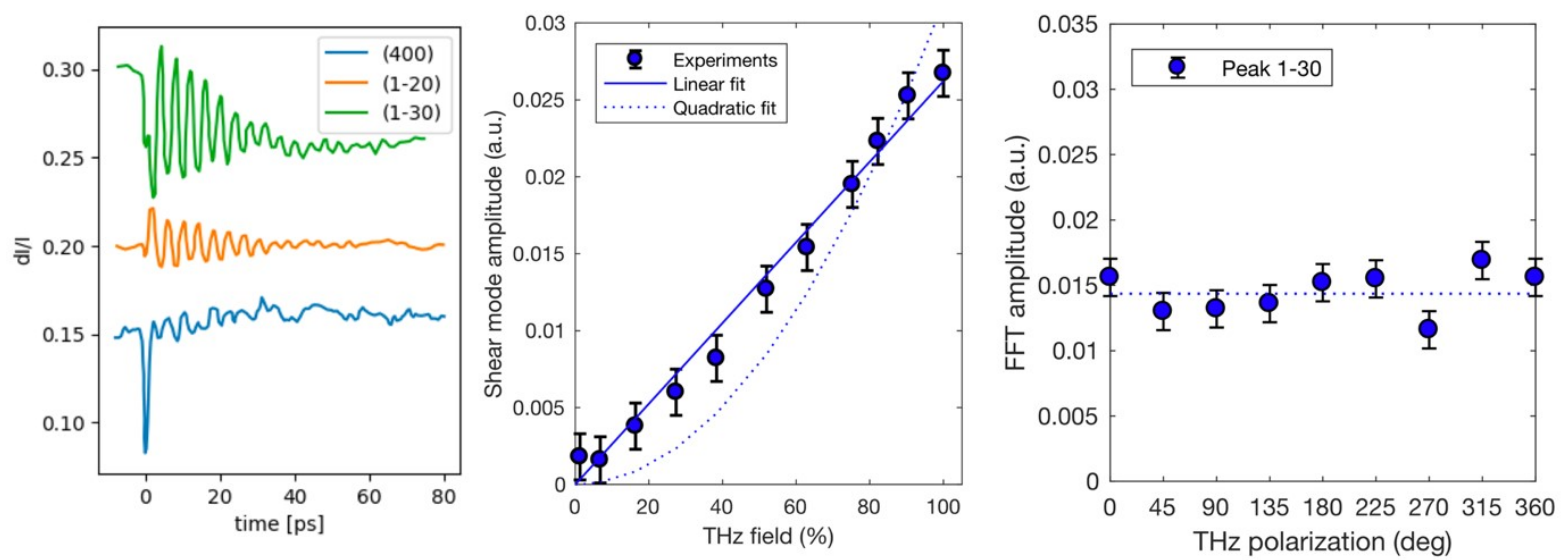

Figure 1. (left) Time-dependence of various reflections indexed by their miller indices induced by single-cycle $\mathrm{THz}$ pulses (center frequency $1 \mathrm{THz}$ ). Measurement of many reflections such as these identifies the crystallographic direction and structural deformation that is induced, corresponding to shear motion along the [ $\left[\begin{array}{lll}0 & -1 & 0\end{array}\right]$ axis, towards that of $1 \mathrm{~T}^{\prime} \mathrm{WTe}_{2}$. (middle) Shear mode amplitude as a function of applied $\mathrm{THz}$ field. (right). Shear mode amplitude as the $\mathrm{THz}$ polarization is rotated through 360 degrees indicating an effect which does not depend on the sign of the applied E-field. 